\title{
EGFP-EGFI-conjugated poly(lactic-co-glycolic acid) nanoparticles, a new diagnostic tool and drug carrier for atherosclerosis
}

This article was published in the following Dove Medical Press journal: International Journal of Nanomedicine

\author{
Zhilin $\mathrm{Wu}^{\prime}$ \\ Chen Chen' \\ Bo Zhang ${ }^{2}$ \\ Liang Tang ${ }^{2}$ \\ Wei Shi \\ Danying Liao ${ }^{2}$ \\ Gaohong $\mathrm{Di}^{\prime}$ \\ Jacques RJ Davis' \\ Hui Wang
}

'Anesthesiology Department, Union Hospital, Tongji Medical College, Huazhong University of Science and Technology, Wuhan 430022, People's Republic of China; ${ }^{2} \mathrm{Haematology}$ Department, Union Hospital, Tongji Medical College, Huazhong University of Science and Technology, Wuhan 430022, People's Republic of China
Correspondence: Chen Chen Anesthesiology Department, Union Hospital, Tongji Medical College, Huazhong University of Science and Technology, Wuhan 430022, People's Republic of China Email 33465909।@qq.com
Background: EGFP-EGF1-conjugated poly(lactic-co-glycolic acid) (PLGA) nanoparticle (ENP) has a specific affinity to tissue factor (TF). The aim of this study was to investigate the target delivery of ENP to plaques and its uptake in a mouse model of atherosclerosis in vivo and in vitro. Materials and methods: Coumarin-6- and 1,1'-dioctadecyl-3,3,3',3'-tetramethylindotricarbo cyanine iodide (DiR)-loaded ENPs were synthesized using a double-emulsion method. Mouse vascular smooth muscle cells (VSMCs) were induced with MCP-1 to obtain an increased TF expression. Fluorescence microscopy and flow cytometry assay were performed to examine the uptake of coumarin-6-loaded ENPs in cellular models. An animal model of atherosclerosis was established with an ApoE (-/-) mouse fed with continuous high-fat diets for 14 weeks. DiR-loaded ENPs (DiR-ENPs) were injected via the caudal vein. The distribution of DiR-ENPs was examined through organ imaging and confocal laser scanning microscopy.

Results: Results indicated TFs were highly expressed in the cellular model. The uptake of coumarin-6-loaded ENPs was significantly higher than that of common PLGA nanoparticles. Thickening of intima and lipid deposition in the aorta could be observed in atherosclerosis mouse models. Confocal laser scanning microscopy organ imaging showed ENPs accumulated in vessels with atherosclerotic plaques, which coincided with high expressions of TF.

Conclusion: This study showed that EGFP-EGF1-conjugated PLGA nanoparticles could be effectively delivered to atherosclerotic plaques in vivo and taken up by VSMCs with high TF expressions in vitro. Thus, it could be a promising carrier for targeted therapy of atherosclerosis. Keywords: tissue factor, atherosclerosis, nanoparticle, target delivery, EGFP-EGF1

\section{Introduction}

Atherosclerosis is an inflammatory disease that leads to the hardening and thickening of the arterial walls and subsequently results in the formation of plaques, which are comprised of immune cells, mesenchymal cells, lipids, and extracellular matrix. ${ }^{1-3}$ It is a predominant cause of a variety of cardiovascular disorders including myocardial infarction, ischemic stroke, and peripheral vascular disease and accounts for significant morbidity and mortality rates in the aged population. ${ }^{4,5}$ Atherosclerosis is initiated by the dysfunction of endothelial cells. ${ }^{6,7}$ The vicious cycle promotes the proliferation of vascular smooth muscle cells (VSMCs) and reconstruction of extracellular matrix, ultimately leading to the formation of atherosclerotic plaques. ${ }^{8,9}$

Tissue factor (TF), a key molecular component in initiating the external coagulation pathway, has been reported to increase in the plasma of patients with myocardial infarction and hyperlipidemia. ${ }^{10,11}$ TF participates in nearly all pathological processes of atherosclerosis. ${ }^{12}$ Inflammatory mediators such as monocyte chemoattractant 
protein-1 (MCP-1) are secreted by activated monocytes/ macrophages inducing more TF expression in VSMCs ${ }^{13,14}$ and endotheliums. ${ }^{15}$ As the disease progresses, multiple TFs can be detected in the lipid core and extracellular matrix of plaques. Increased expression of TF mediates the migration and proliferation of smooth muscle cells into the intima of arteries, promoting angiogenesis, vascular reconstruction, and weakening of the stability of plaques. ${ }^{16}$ As unstable plaques rupture, foam cells and micro-particles with high $\mathrm{TF}$ contents are released from the plaque, resulting in a hypercoagulable state and thrombosis-related complications such as acute myocardial infarction. ${ }^{17,18}$

There are generally no symptoms in the early stages of atherosclerosis. Traditional detection techniques cannot easily, safely, and effectively detect the lesions in the early stages. In recent years, there has been a rapid development in using nanoparticle (NP) technique as a tool for molecular imaging of atherosclerotic lesion. ${ }^{19-21}$ By incorporating peptides, antibodies, or other ligands on its surface, a NP can target adhesion molecules in lesion components. ${ }^{22}$ Engineered supramolecular micelles targeting cellular components in atherosclerotic plaques, such as monocytes, exhibit promising characteristics for the diagnosis of the disease. ${ }^{23,24}$ In our previous research, we developed a novel fusion protein EGFP-EGF1, which is synthesized based on the TF gene in the EGF1 region and has a specific affinity to $\mathrm{TF}^{25}$ It has been proved that EGFP-EGF1-poly(lactic-co-glycolic acid) (PLGA) NPs can mediate target delivery of siRNA to injured brain microvascular endothelial cells (BMECs) in rats. ${ }^{26} \mathrm{As}$ TF is significantly expressed in macrophages and VSMCs in atherosclerotic lesions, it is hypothesized that EGFP-EGF1-PLGA NPs (ENPs) could also be delivered to atherosclerotic plaques by virtue of its specific binding ability to TF, and thus could be utilized as a tool for imaging and target treatment of the disease. The purpose of this study was to evaluate the distribution of ENPs in a mouse atherosclerosis model in vivo and their uptake in cultured VSMCs in vitro.

\section{Methods}

\section{Materials and animals}

The Escherichia coli strain BL21 (DE3) and plasmid pET28a-EGFP-EGF1 were maintained in our laboratory as previously described. ${ }^{25}$ PLGA (50:50, inherent viscosity of 0.89 , MW $100 \mathrm{kDa}$ ) was purchased from Absorbable Polymers (Pelham, AL, USA). Methoxy-poly-(ethylene glycol) (M-PEG, MW 3,000 Da) was purchased from the NOF Co. (lot no 14530; Tokyo, Japan) and maleimide-PEG (Mal-PEG, MW 3,400 Da) was purchased from Nektar Co. (lot no PT-08D-16; Santa Ana, CA, USA). Rabbit polyclonal antibody against rat TF was purchased from Santa Cruz Biotechnology Inc. (Dallas, TX, USA). DMEM (high glucose), RPMI 1640 medium, and FBS were purchased from Thermo Fisher Scientific (Waltham, MA, USA). MCP-1 was purchased from R\&D Biological Co. (Minneapolis, MN, USA). Real-time PCR primers were synthesized by Thermo Fisher Scientific. All other chemicals were analytical reagent grade, and were purchased from the Sino-pharm Chemical Reagent Co. (Hangzhou, China). C57/B background mice with ApoE gene knockout were provided by the Center of Experimental Animals at Peking University (Beijing, China). The animal procedures were approved by the Animal Care Committee of the Tongji Medical College of Huazhong University of Science and Technology and followed the Regulations of the Administration of Affairs Concerning Experimental Animals.

\section{Cell culture and preparation}

\section{In vitro cellular model preparation}

VSMCs were isolated from C57/B mouse (20-25 g) as previously described ${ }^{27,28}$ and cultured in DMEM supplemented with $20 \% \mathrm{FBS}$ at $37^{\circ} \mathrm{C}$ in a humidified atmosphere containing $5 \% \mathrm{CO}_{2}$. TF expression was induced by incubating VSMCs with MCP-1 (10 ng/mL) ${ }^{29}$ for 0, 1, 2, 4, 8, 16, and 24 hours. Then the real-time PCR was performed and the optimal induction time was selected. The primers used for PCR were as follows: TF, sense: 5'-GCACCGAGCAATGGAAGAG-3', antisense: 5'-CAGAGATATGGACAGGAGGATGAT-3'; GAPDH: sense: $5^{\prime}$-ATGGTGGTGAAGACGCCAGTA-3', antisense: 5'-GGCACAGTCAAGGCTGAGAATG-3'.

\section{Preparation of nanoparticles}

The PLGA NPs were prepared using water-in-oil-in-water (W/O/W) double-emulsion solvent evaporation method and conjugated with EGFP-EGF1 fusion protein similar to a previously described protocol. ${ }^{26,30}$ Coumarin-6- or DiR (DiIC18(7); 1,1'-dioctadecyl-3,3,3',3'-tetramethylindotricarbocyanine iodide)-loaded NPs/ENPs were prepared with the same procedure except that $30 \mathrm{mg}$ of coumarin- 6 or $200 \mathrm{mg}$ of DiR was additionally added to dichloromethane containing copolymers before primary emulsification.

\section{Characterization of nanoparticles}

The mean diameter and zeta potential of the NPs were determined by dynamic light scattering (DLS) using the zeta-potential/particle sizer Nicomp 380 ZLS (Particle Sizing Systems, Santa Barbara, CA, USA). The NPs were morphologically examined by transmission electron microscopy (H-600; Hitachi, Tokyo, Japan). 


\section{Establishment of atherosclerosis model in vivo}

ApoE gene knockout mice were fed with high-fat $(0.2 \%$ cholesterol, 21\% fat) diet (HFD) for 14 weeks to establish the classical atherosclerosis model. The control group mice were fed with normal diet (CHD). Oil red O staining of aorta was performed to confirm the development of atherosclerosis. ${ }^{31}$ The mRNA was extracted from the aorta of ApoE (-/-) mice after they were fed with HFD for $0,8,9,10,12$, and 14 weeks. Five mice were included in each group.

\section{Cellular uptake study}

VSMCs were seeded at a density of $1 \times 10^{6}$ per well in sixwell plates and incubated for 20 hours. Then the medium was exchanged with a serum-free medium. VSMCs were treated with MCP-1 (10 ng/mL) for 2 hours. Coumarin6-labeled ENPs and NPs were mixed with cultured cells and incubated for 30 minutes at $37^{\circ} \mathrm{C}$. The cells were washed two times with PBS (0.01 M, pH =7.4) and immobilized with $4 \%$ paraformaldehyde for 20 minutes at room temperature. Then, the fluorescence intensity of the cells was detected by flow cytology and fluorescence microscopy. Untreated cells were incubated with NPs to serve as a control.

\section{Distribution of nanoparticles in vivo}

Mice in the HFD group were randomly divided into three groups with eight mice in each group. They were separately injected with DiR-loaded ENPs (DiR-ENPs, $0.05 \mathrm{mg} / \mathrm{g}$ ), DiR-loaded NPs (DiR-NPs, $0.05 \mathrm{mg} / \mathrm{g}$ ), and PBS $(0.01 \mathrm{M}$, $\mathrm{pH}=7.4)$ via the caudal vein. Mice in the CHD group were injected with DiR-ENP $(0.05 \mathrm{mg} / \mathrm{g})$ to serve as a control. One hour later, the mice were sacrificed. Then, the fluorescence images of the organs were obtained by placing the tissue in a living-body imager, and the relative light intensity of each organ was obtained.

\section{Distribution of nanoparticles in aorta}

Organ imaging and immune fluorescence microscopy examination of aorta were performed. Then frozen sections from the specimen were incubated with TF antibody $(1: 100)$ for $4^{\circ} \mathrm{C}$ after $5 \%$ BSA closure. Then CY3-labeled secondary antibody (1:100) was added to the sample at room temperature for 30 minutes after washing. The nuclei were dyed with DAPI. Finally, the aortal specimens were observed under a confocal laser microscope.

\section{Statistical analysis}

Statistical analysis was performed using SPSS 18.0 (SPSS Inc., Chicago, IL, USA). Data were presented as mean \pm standard error of mean (SEM). Statistical comparisons between the two groups were evaluated by Student's $t$-test and one-way ANOVA was used for more than two groups. A value of $P<0.05$ was considered to indicate a statistically significant difference.

\section{Results}

\section{Characteristics of nanoparticles}

The PLGA NPs were prepared using double-emulsion solvent evaporation method and conjugated with the EGFP-EGF1 fusion protein. After coumarin-6 or DiR was loaded into the NPs, DLS showed the mean size of coumarin-6 and DiRloaded NPs/ENPs to be about $100 \mathrm{~nm}$ (Table 1, Figure 1). Their zeta potential was around $-11 \mathrm{mV}$ (Table 1).

\section{Establishment of cellular model}

To establish cellular models of atherosclerosis, VSMCs were induced with MCP-1 (10 ng/mL). TF molecules were expressed in the cultured cellular model as in an atherosclerotic plaque. Real-time PCR was used to investigate the relationship between time and TF expression levels. TF mRNA reached its peak after a 2-hour exposure to MCP-1 in VSMCs (Figure 2). Thus, VSMCs were induced with MCP-1 for 2 hours to establish a cellular model of atherosclerosis for in vitro NPs research.

\section{Fluorescence microscopy examination to determine nanoparticle uptake}

VSMCs were exposed to MCP-1 (10 ng/mL) for 2 hours. Then the cellular model was incubated with coumarin6-loaded ENPs and NPs. Under a fluorescence microscope, a much stronger fluorescence could be observed in the ENP group than in the NP group (Figure 3).

\section{Flow cytometry to determine nanoparticle uptake}

VSMCs were exposed to MCP-1 (10 ng/mL) for 2 hours. Then they were incubated with coumarin-6-loaded ENPs and NPs. Flow cytometry was performed for the quantitative analysis of the fluorescence intensity. It revealed that fluorescence

Table I Particle size and zeta potential of nanoparticles

\begin{tabular}{|l|l|l|}
\hline Nanoparticles & Mean size $(\mathbf{n m})$ & Zeta potential $(\mathbf{m V})$ \\
\hline Coumarin-6-ENP & $102.06 \pm 3.32$ & $-11.61 \pm 2.01$ \\
\hline Coumarin-6-NP & $96.86 \pm 2.42$ & $-11.71 \pm 2.98$ \\
\hline DiR-ENP & $103.08 \pm 2.73$ & $-11.15 \pm 2.19$ \\
\hline DiR-NP & $95.65 \pm 3.56$ & $-11.23 \pm 2.73$ \\
\hline
\end{tabular}

Note: Measured in double-distilled water $(n=3)$, mean $\pm S D$.

Abbreviations: ENP, EGFP-EGFI-conjugated poly(lactic-co-glycolic acid) nanoparticle; NP, nanoparticle; DiR, I,I'-dioctadecyl-3,3,3',3'-tetramethylindotricarbocyanine iodide. 


$\begin{array}{rllll} & & \text { Size }(\mathbf{d} \cdot \mathbf{n m}) & \% \text { intensity } & \text { Width }(\mathbf{d} \cdot \mathbf{n m}) \\ \text { Z-average }(\mathrm{d} \cdot \mathrm{nm}): \mathbf{1 0 1 . 4} & \text { Peak 1: } & 119.4 & 100 & 48.33 \\ \text { Pdl: } \mathbf{0 . 1 4 2} & \text { Peak 2: } & 0.000 & 0.0 & 0.000 \\ \text { Intercept: } \mathbf{0 . 9 6 7} & \text { Peak 3: } & 0.000 & 0.0 & 0.000\end{array}$

Result quality: good

\section{Size distribution by intensity}

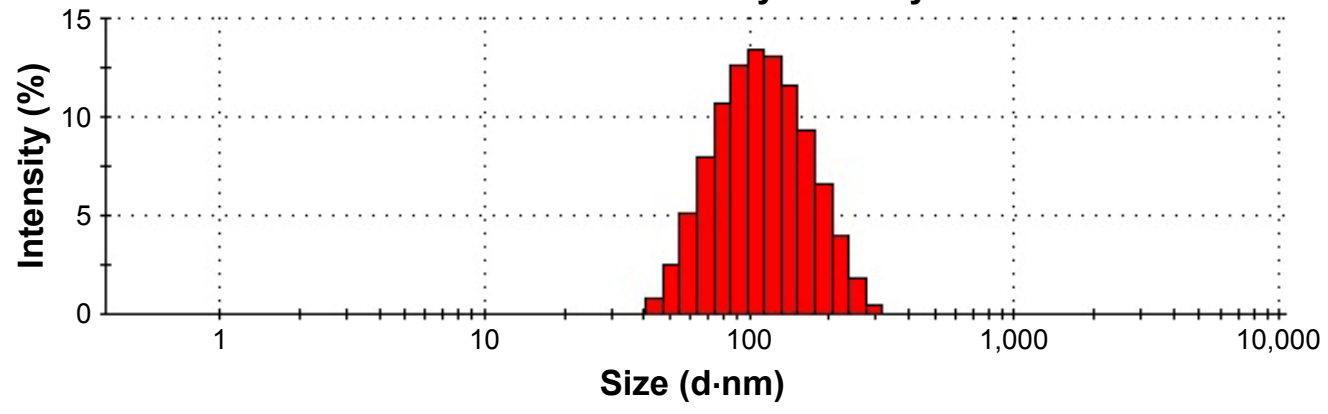

Figure I Particle size distribution of coumarin-6-labeled ENP.

Abbreviation: ENP, EGFP-EGFI-conjugated poly(lactic-co-glycolic acid) nanoparticle.

intensity in the ENPs group was significantly higher than in the NPs group (Figure 4), suggesting that ENPs were more easily taken up by the cells than NPs.

\section{Identification of mouse atherosclerosis model}

Male ApoE gene knockout mice, aged 3-4 weeks with a body weight of 20-25 g, were fed with HFD continuously

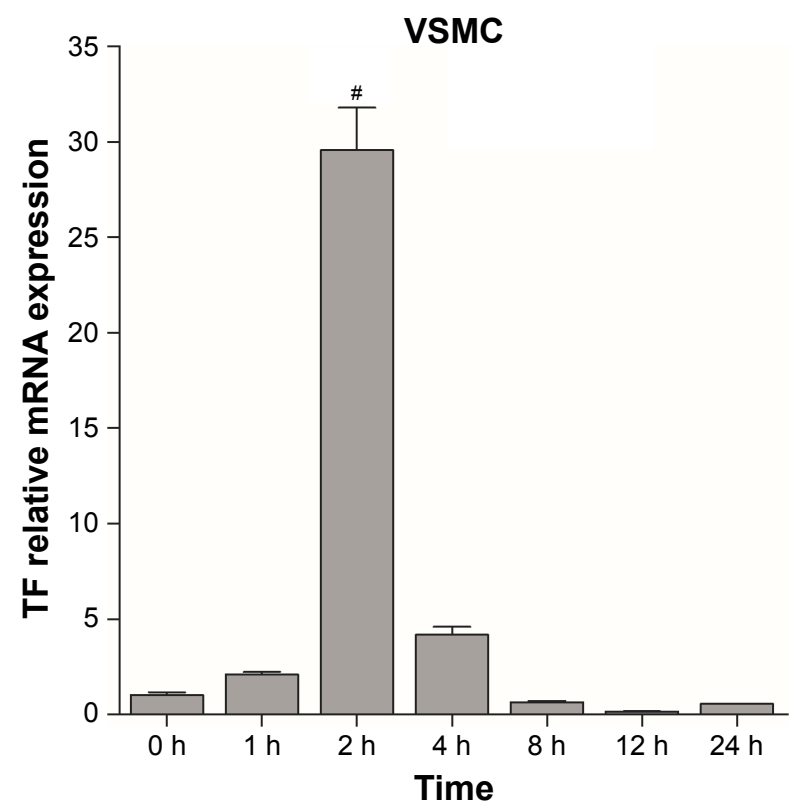

Figure 2 TF expression in VSMCs at different timepoints. The experiments were performed in triplicate to calculate the average value of each group. Data are presented as mean \pm SEM. The TF mRNA levels from the different treated cells were normalized to the control one; ${ }^{\#}<<0.05$.

Abbreviations: TF, tissue factor; VSMCs, vascular smooth muscle cells; SEM, standard error of mean; $h$, hours. for 14 weeks. Oil red $\mathrm{O}$ staining of the aorta was done to confirm the development of atherosclerosis. Microscopy showed that the intima of the aorta was thickened and reddish brown lipid was deposited on the arterial wall (Figure 5A). These findings proved the successful establishment of the mouse atherosclerosis model. Mice were sacrificed at different week intervals after they were fed with HFD. The tissue of aorta was harvested, and real-time PCR revealed that TF expression reached its peak after feeding HFD for 14 weeks (Figure 5B).

\section{Distribution of nanoparticles in mouse atherosclerosis model}

The fluorescence signal of DiR-ENP group was significantly stronger than that of DiR-NP group, especially at the aortic arch (Figure 6A). The fluorescence signals were collected from other major organs of the animal. It could be clearly observed that the NPs were highly aggregated in the liver, spleen, and lung of mice after exposure for a brief period. There was no significant difference between the two groups, but the uptake of Dir-ENPs in the aortic region of atherosclerotic plaques was significantly higher than that of Dir-NPs, and the fluorescence signal was found to be enhanced by about 1.73 times (Figure 6B).

\section{Distribution of DiR-ENPs in aorta of mouse atherosclerosis model}

After the injection of NPs, the mouse model was sacrificed and a slice of aorta tissue was examined under a fluorescence microscope (Figure 7). Tissues with DiR-ENP deposition 

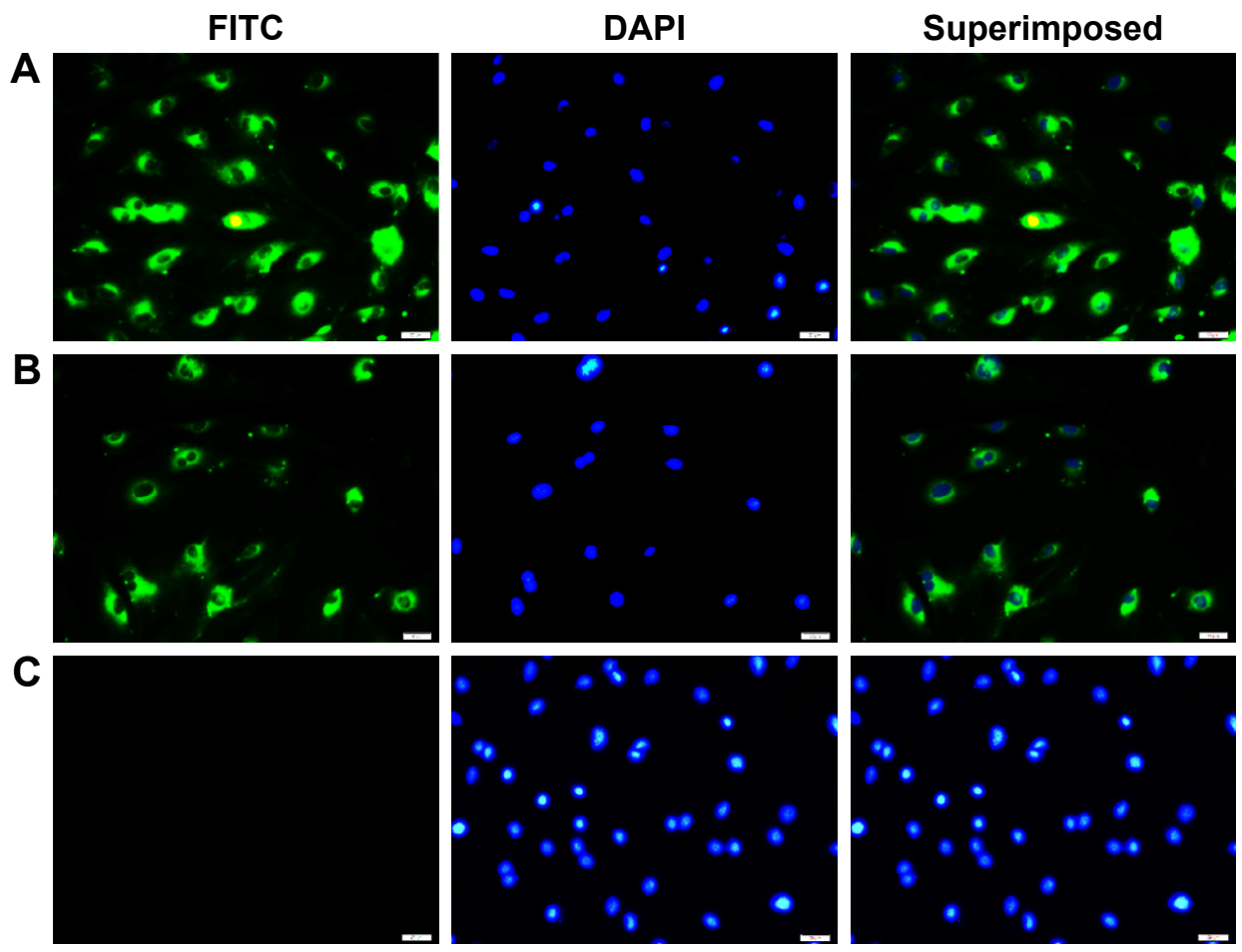

Figure 3 Uptake of coumarin-6 nanoparticles by stimulated VSMCs.

Notes: (A) Shows the ENP group of cells. (B) Shows the NP group of cells. (C) Shows cells incubated with PBS only (0.0I M, pH 7.4). Nuclei are stained with DAPI (blue). Scale bar: $100 \mathrm{~nm}$.

Abbreviations: VSMCs, vascular smooth muscle cells; ENP, EGFP-EGFI-conjugated poly(lactic-co-glycolic acid) nanoparticle; NP, nanoparticle; FITC, fluorescein isothiocyanate.

exhibited green fluorescence and tissues with high TF expression exhibited red fluorescence. It could be observed that more TFs were expressed in the aorta of mice fed with HFD (Figure 7A-C) than in mice fed with normal diet (Figure 7B). ENPs preferentially accumulated in the tissues exhibiting TF expression (Figure 7A). Only a small amount of ENPs was taken up by the mice fed with a normal diet
(CHD) (Figure 7B). No NP deposition could be observed in the aorta of the mouse atherosclerosis model following DiR-NP injection (Figure 7C).

\section{Discussion}

Current preventive and therapeutic modalities of atherosclerosis focus on improving blood lipid profile, inhibiting

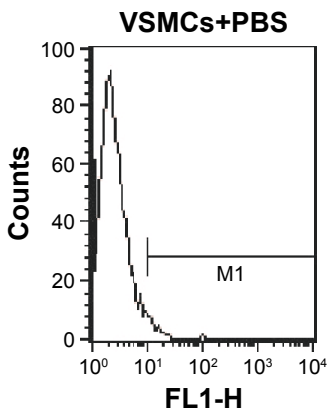

Marker \% gated Mean Geo mean

$\begin{array}{llll}\text { All } & 100 & 2.77 & 2.37\end{array}$

$\begin{array}{llll}\text { M1 } & 1.62 & 12.95 & 12.37\end{array}$

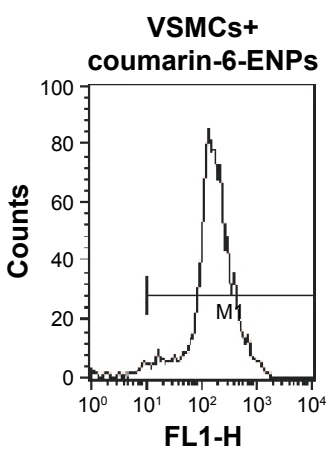

Marker \% gated Mean Geo mean

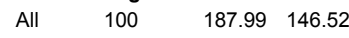

$\begin{array}{llll}\text { M1 } & 99.05 & 189.72 & 150.86\end{array}$
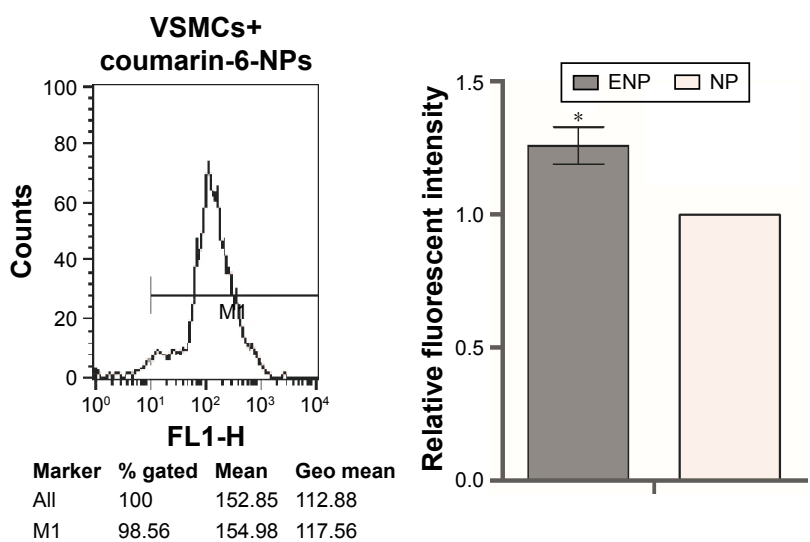

Figure 4 Cell fluorescence intensity of VSMCs.

Notes: The experiment was repeated three times to calculate the average fluorescence intensity of each group, and the histogram was obtained based on NP group. Data are presented as mean $\pm S E M ; * P=0.001$.

Abbreviations: VSMCs, vascular smooth muscle cells; NP, nanoparticle; SEM, standard error of mean; ENP, EGFP-EGFI-conjugated poly(lactic-co-glycolic acid) nanoparticle; Geo, geometric. 

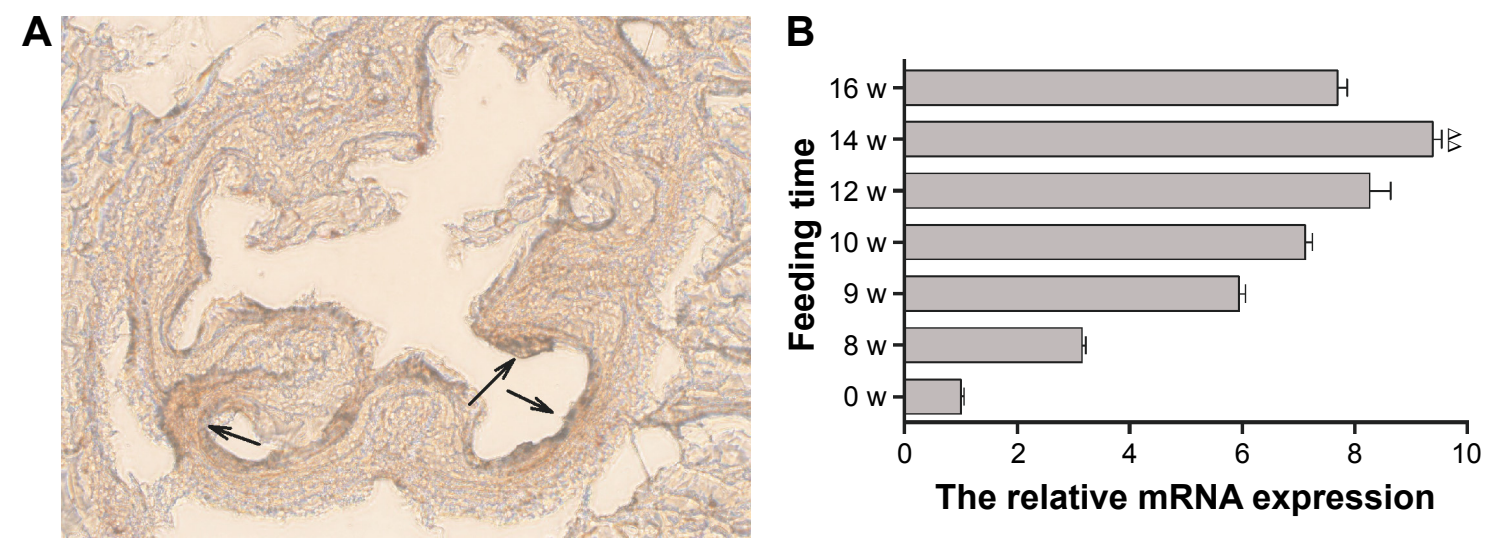

Figure 5 (A) Oil red $O$ staining of frozen sections of the aorta, arrowhead showing lipid deposition on the arterial wall. (B) TF mRNA expressions in aorta of mouse atherosclerosis models fed with high-fat diets for various lengths of time. Data are presented as mean $\pm \mathrm{SEM} ; \triangle \triangle P<0.05$.

Abbreviations: TF, tissue factor; SEM, standard error of mean; w, weeks.

thrombus formation, and decreasing blood pressure, but the treatment cannot directly target specific molecules, cells, and processes involved in the formation of atherosclerotic lesions. ${ }^{32}$ PEG-PLGA NPs have been used as drug carriers due to their physical stability and property of sustained drug release, which is believed to be suitable for the treatment of chronic diseases, and hence is approved by US FDA. ${ }^{33,34}$ In our previous research, we established a stable EGFPEGF1-PLGA NP with a specific affinity to TF. The NPs could be delivered to injured BMECs, neuroglial cells, and glioma cells and can be used as a carrier for target therapy. ${ }^{26,35}$

Numerous studies have shown that TF expression increased significantly in atherosclerosis patients. TF plays a key role in the pathogenesis of the disease. ${ }^{10,36,37}$ If ENP could be target-delivered to atherosclerotic lesions by binding with $\mathrm{TF}$, it would serve as an ideal carrier for therapeutic agents, thereby achieving a controlled and target delivery of the drug with better efficacy and fewer side effects.
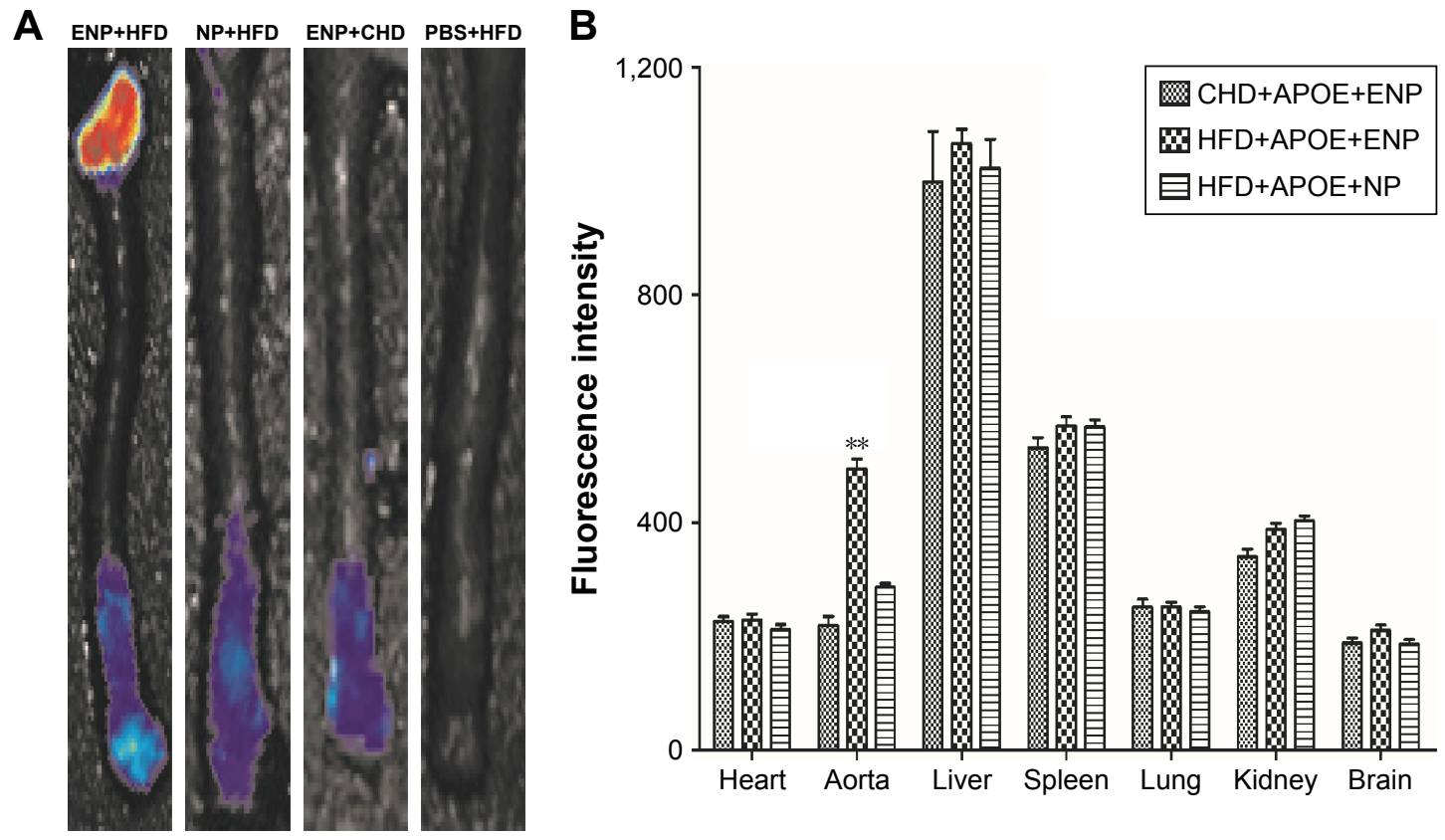

Figure 6 Organ imaging of DiR-ENPs in atherosclerosis mice.

Notes: (A) Shows the fluorescence imaging of the mouse aorta. (B) Shows the fluorescence intensity of different organs, and the histogram was analyzed by statistical analysis. Data are presented as mean \pm SEM ( $n=6$ per group); $* * P<0.05$.

Abbreviations: DiR, I, I'-dioctadecyl-3,3,3',3'-tetramethylindotricarbocyanine iodide; ENPs, EGFP-EGFI-conjugated poly(lactic-co-glycolic acid) nanoparticle; SEM, standard error of mean; NP, nanoparticle; HFD, high-fat diet; CHD, normal diet. 

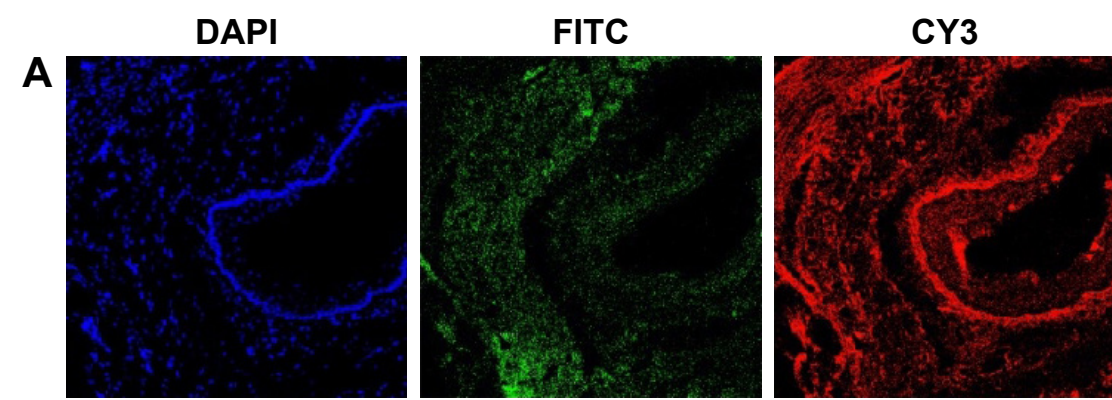

\section{Superimposed}

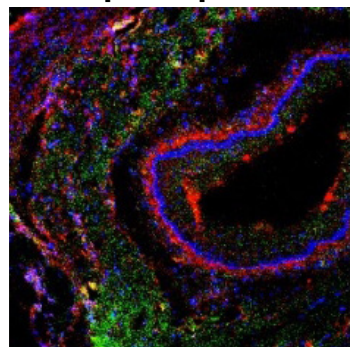

B
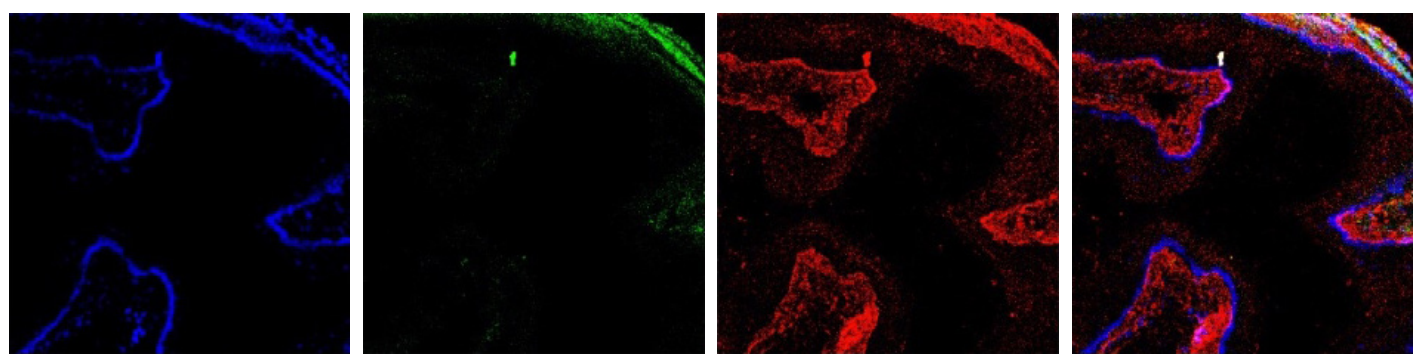

C

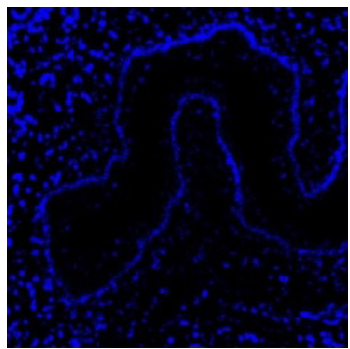

D

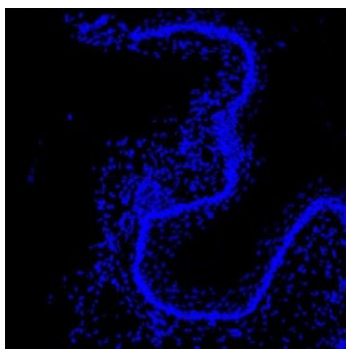

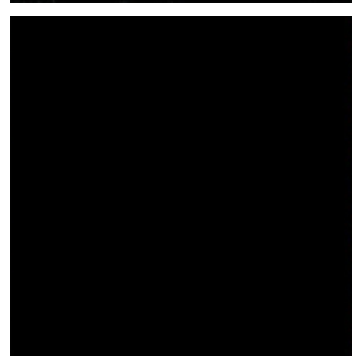

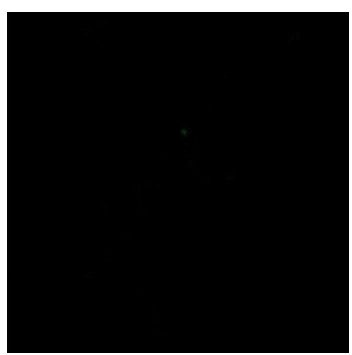

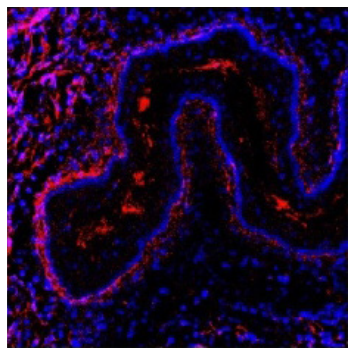
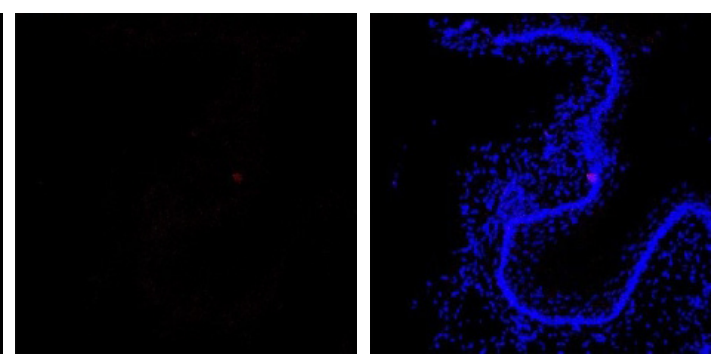

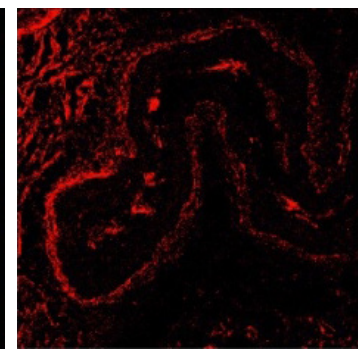

Figure 7 Laser confocal observation of the distribution of nanoparticles in the aorta of atherosclerosis model mice.

Notes: (A) Shows the aorta after injection of DiR-ENPs into the atherosclerosis mouse model of HFD group. (B) Shows the aorta after injection of DiR-ENPs in CHD group. (C) Shows the aorta after injection of DiR-NPs in HFD group. (D) is the aorta after PBS injection in HFD group, no incubation with TF antibody. FITC-labeled EGFP-EGF I site is shown in green and CY3-labeled TF expression site in red. Nuclei were stained with DAPI (blue).

Abbreviations: DiR, I,I'-dioctadecyl-3,3,3',3'-tetramethylindotricarbocyanine iodide; ENPs, EGFP-EGFI-conjugated poly(lactic-co-glycolic acid) nanoparticle; TF, tissue factor; HFD, high-fat diet; CHD, normal diet; FITC, fluorescein isothiocyanate.

VSMCs are one of the main TF resources and contributing cell types for the formation of atherosclerotic plaque. ${ }^{38}$ MCP-1 is the main inducer of TF expression in VSMCs. Through the FVIIa receptor pathway, TF promotes migration and proliferation of VSMCs into the intima of arteries, leading to vessel injury and vascular reconstruction. ${ }^{10,39}$ Based on their involvement in the formation of atherosclerotic plaque, tests to determine the uptake of ENPs were performed in VSMCs.

MCP-1 was used to induce TF expression in VSMCs. TF mRNA in cultured VSMCs reached its peak after being stimulated with MCP-1 for 2 hours. Coumarin-6, a fluorescent marker, was used for tracing the uptake of ENPs in cultured cells. Both fluorescence microscopy and flow cytometry results proved that more ENPs were taken up than NPs in the cellular model. The results strongly suggested that EGFPEGF1-NPs could specifically bind to the EGF1 receptors, ie, TF, on the surface of cultured VSMCs.

The structure of atherosclerotic plaques includes a fibrin cap, a large lipid (primarily cholesterol) core, proliferated smooth muscle cells, and remodeled extracellular matrix. ${ }^{16}$ The dense fibrin cap may obstruct the entry of ENP into the plaques. To further confirm the in vitro findings of this 
study with regard to specificity and sensitivity of ENPs in atherosclerosis, DiR, a near-infrared dye, was employed as a tag for ENPs. ApoE (-/-) mice were the rodents of choice for establishing the animal atherosclerosis model. After continuous feeding of HFD, the animal models exhibited symptoms similar to human atherosclerosis disease. Oil red staining revealed plaque formation in the aorta, and the serum cholesterol and lipid levels were found to be markedly elevated. All these observations proved the successful establishment of atherosclerosis models.

The fusion protein, EGFP-EGF1, was synthesized based on the TF gene in rats. Based on the fact that the similarity of EGF1 region between rats and mice is $95 \%$, we assume that the affinity of EGFP-EGF1 to TF molecule in mice is similar to that of rat. To study the distribution of NPs in vivo, DiR-ENPs were administered via the caudal vein of the atherosclerotic animal. By organ imaging technique, it could be observed that there was no statistical difference with regard to fluorescence intensity in the liver, spleen, kidney, brain, and lungs among the three groups. It showed that the distribution of NPs in these organs was nonspecific, which was determined by blood flow to the organs. However, in the aorta, there was a significant statistical difference between DiR-ENPs group and DiR-NPs group $(P<0.05)$. It indicated that ENPs have a specific affinity to the plaque in a mouse atherosclerosis model.

To further confirm the relationship between the distribution of ENPs and expression of TF in plaques, fluorescence-labeled TF antibody CY3 was cocultured with the aortic tissue. Confocal laser scanning microscopy was employed, and the results indicated that TF was significantly expressed at the sites in the aorta where ENPs were highly accumulated. It suggested that ENPs entered into the plaque through dysfunctional endothelial layer or penetrated through the unstable fibrin cap of plaques, via binding with TF molecules on the cellular surface. VSMC is one of the targeting cell types as it is one of the main resources for TF in atherosclerosis. By a mechanism of ligand-receptor-mediated endocytosis, the ENPs were taken up into the cytoplasm of the cells constituting the plaques.

Diagnostic dyes or contrast can be incorporated into NPs, which can be detected using modalities including magnetic resonance imaging, positron emission tomography/single photon emission computed tomography, computed tomography, and optical near-infrared fluoroscopy. ${ }^{40}$ Our study proved that ENPs specifically and effectively bind to plaques. If ENPs are incorporated with the imaging agents, they will be useful for the diagnosis of atherosclerosis. Imaging of specific cells or components in lesions can disclose lesion biology and features, especially vulnerability, which can help prevent and treat major cardiovascular events. Thus, ENPs can also work as a tool for target therapy of atherosclerosis by providing specific medication, siRNA, etc, to decrease the inflammation, inhibit progression, and stabilize the plaques. Studies showed that TF is expressed by dysfunctional endothelial cells, VSMCs, and monocyte/macrophages at the early stage of atherosclerosis. Compared with other molecular NPs, which target single cellular type of atherosclerosis, ENPs could not only be taken up by VSMCs, a major cellular component of atherosclerotic plaques, but there is also a high possibility that ENPs could be taken up by other cell types such as dysfunctional endothelial cells and monocytes in atheromatous plaque, as TFs are also expressed in these cells. Based on the high affinity and sensitivity of the NP to TF protein, ENPs could also be beneficial for early imaging and prevention of atherosclerosis.

However, there are some limitations in our research. In vivo distribution of NPs was studied for just 1 hour after the administration. This short time period may be adequate for diagnostic purposes. However, to work as an effective drug carrier for use in targeting therapy, the information we obtained from the research is obviously not enough. Clotting and other conditions associated with abnormally high TF expression may affect the distribution and drug delivery of the NP. As macrophages/foam cells in atherosclerotic plaques are TF-rich cellular types, they might also contribute to the imaging results after ENP administration. Hence, the uptake of ENPs by these cells should also be studied. With respect to these findings, some questions need to be answered: Can these particles remain in the localized lesions for longer time? Will these NPs support targeted drug delivery in vivo? Is there any systemic effect on a healthy body after ENP administration? Further research needs be done to obtain more information about ENP distribution and their systemic effect in atherosclerosis, especially their value as a promising carrier for targeting prevention strategies and therapy for atherosclerosis needs to be studied.

In summary, this research proved that the EGFP-EGF1conjugated NP could be effectively taken up by VSMCs in the atherosclerosis cellular model and be delivered into the sites of atherosclerotic plaque in vitro and in vivo. This NP presents a promising advantage for target drug delivery in diagnosis and therapy of atherosclerosis.

\section{Acknowledgments}

This work was supported by the National Natural Science Foundation of China (Nos 81500342 and 81600175). The funder had no role in study design, data collection and analysis, decision to publish, or preparation of the manuscript. 


\section{Author contributions}

$\mathrm{CC}$ contributed to the conception and design of this study. ZW contributed to data acquisition. WS, BZ, and DL carried out animal experiments. GD and HW contributed to the analysis and interpretation of the data. JRJD and LT drafted the manuscript and critically revised it for important intellectual content. All authors contributed to data analysis, drafting and revising the article, gave final approval of the version to be published, and agree to be accountable for all aspects of the work.

\section{Disclosure}

The authors report no conflicts of interest in this work.

\section{References}

1. Weber C, Noels H. Atherosclerosis: current pathogenesis and therapeutic options. Nat Med.2011;17(11):1410-1422. doi:10.1038/nm.2538

2. Feig JE, Parathath S, Rong JX, et al. Reversal of hyperlipidemia with a genetic switch favorably affects the content and inflammatory state of macrophages in atherosclerotic plaques. Circulation. 2011;123(9): 989-998. doi:10.1161/CIRCULATIONAHA.110.984146

3. Williams KJ, Feig JE, Fisher EA. Rapid regression of atherosclerosis: insights from the clinical and experimental literature. Nat Clin Pract Cardiovasc Med. 2008;5(2):91-102. doi:10.1038/ncpcardio1086

4. Weber C, Zernecke A, Libby P. The multifaceted contributions of leukocyte subsets to atherosclerosis: lessons from mouse models. Nat Rev Immunol. 2008;8(10):802-815. doi:10.1038/nri2415

5. Libby P. Inflammation in atherosclerosis. Nature. 2002;420(6917): 868-874. doi: $10.1038 /$ nature 01323

6. Min K, Cho K, Kwon TK. The effect of oxidized low density lipoprotein (oxLDL)-induced heme oxygenase-1 on LPS-induced inflammation in RAW 264.7 macrophage cells. Cell Signal. 2012;24(6):1215-1221. doi:10.1016/j.cellsig.2012.02.001

7. Tsimikas S, Miller YI. Oxidative modification of lipoproteins: mechanisms, role in inflammation and potential clinical applications in cardiovascular disease. Curr Pharm Des. 2011;17(1):27-37.

8. Tabas I. Macrophage death and defective inflammation resolution in atherosclerosis. Nat Rev Immunol. 2010;10(1):36-46. doi:10.1038/ nri2675

9. Moore KJ, Sheedy FJ, Fisher EA. Macrophages in atherosclerosis: a dynamic balance. Nat Rev Immunol. 2013;13(10):709-721. doi:10.1038/ nri3520

10. Tatsumi K, Mackman N. Tissue factor and atherothrombosis. J Atheroscler Thromb. 2015;22(6):543-549. doi:10.5551/jat.30940

11. Steffel J. Tissue factor in cardiovascular diseases: molecular mechanisms and clinical implications. Circulation. 2006;113(5):722-731. doi:10.1161/CIRCULATIONAHA.105.567297

12. Mackman N. Role of tissue factor in hemostasis, thrombosis, and vascular development. Arterioscler Thromb Vasc Biol. 2004;24(6): 1015-1022. doi:10.1161/01.ATV.0000130465.23430.74

13. Schecter AD, Rollins BJ, Zhang YJ, et al. Tissue factor is induced by monocyte chemoattractant protein-1 in human aortic smooth muscle and THP-1 cells. J Biol Chem. 1997;272(45):28568-28573.

14. Schepers A, Eefting D, Bonta PI, et al. Anti-MCP-1 gene therapy inhibits vascular smooth muscle cells proliferation and attenuates vein graft thickening both in vitro and in vivo. Arterioscler Thromb Vasc Biol. 2006;26(9):2063-2069. doi:10.1161/01.ATV.0000235694. 69719.e2

15. Niu J, Kolattukudy PE. Role of MCP-1 in cardiovascular disease: molecular mechanisms and clinical implications. Clin Sci (Lond). 2009; 117(3):95-109. doi:10.1042/CS20080581

16. Libby P. Inflammation in Atherosclerosis. Arterioscler Thromb Vasc Biol. 2012;32(9):2045-2051. doi:10.1161/ATVBAHA.108.179705
17. Li S, Chen H, Ren J, et al. MicroRNA-223 inhibits tissue factor expression in vascular endothelial cells. Atherosclerosis. 2014;237(2): 514-520. doi:10.1016/j.atherosclerosis.2014.09.033

18. ten Cate H. Tissue factor-driven thrombin generation and inflammation in atherosclerosis. Thromb Res. 2012;129(Suppl 2):S38-S40. doi:10.1016/j.thromres.2012.02.028

19. Scharlach C, Kratz H, Wiekhorst F, et al. Synthesis of acid-stabilized iron oxide nanoparticles and comparison for targeting atherosclerotic plaques: evaluation by MRI, quantitative MPS, and TEM alternative to ambiguous Prussian blue iron staining. Nanomedicine-Uk. 2015;11(5):1085-1095. doi:10.1016/j.nano.2015.01.002

20. Ye K, Qin J, Peng Z, et al. Polyethylene glycol-modified dendrimerentrapped gold nanoparticles enhance CT imaging of blood pool in atherosclerotic mice. Nanoscale Res Lett. 2014;9(1):529. doi:10.1186/ 1556-276X-9-529

21. Noukeu LC, Wolf J, Yuan B, Banerjee S, Nguyen KT. Nanoparticles for detection and treatment of peripheral arterial disease. Small. 2018; 1800644. doi:10.1002/smll.201800644

22. Nie S, Zhang J, Martinez-Zaguilan R, et al. Detection of atherosclerotic lesions and intimal macrophages using CD36-targeted nanovesicles. $J$ Control Release. 2015;220(Pt A):61-70. doi:10.1016/j.jconrel. 2015.10.004

23. Mlinar LB, Chung EJ, Wonder EA, Tirrell M. Active targeting of early and mid-stage atherosclerotic plaques using self-assembled peptide amphiphile micelles. Biomaterials. 2014;35(30):8678-8686. doi:10.1016/j.biomaterials.2014.06.054

24. Chung EJ, Mlinar LB, Nord K, et al. Monocyte-targeting supramolecular micellar assemblies: a molecular diagnostic tool for atherosclerosis. $A d v$ Healthc Mater. 2015;4(3):367-376. doi:10.1002/adhm.201400336

25. Mei H, Shi W, Pang Z, et al. EGFP-EGF1 protein-conjugated PEG-PLA nanoparticles for tissue factor targeted drug delivery. Biomaterials. 2010;31(21):5619-5626. doi:10.1016/j.biomaterials.2010.03.055

26. Chen C, Mei H, Shi W, et al. EGFP-EGF1-conjugated PLGA nanoparticles for targeted delivery of siRNA into injured brain microvascular endothelial cells for efficient RNA interference. PLoS One. 2013; 8(4):e60860. doi:10.1371/journal.pone.0060860

27. Ellmark SH, Dusting GJ, Fui MN, Guzzo-Pernell N, Drummond GR. The contribution of Nox 4 to NADPH oxidase activity in mouse vascular smooth muscle. Cardiovasc Res. 2005;65(2):495-504. doi:10.1016/j. cardiores.2004.10.026

28. Sakata Y, Xiang F, Chen Z, et al. Transcription factor CHF1/ Hey2 regulates neointimal formation in vivo and vascular smooth muscle proliferation and migration in vitro. Arterioscler Thromb Vasc Biol. 2004;24(11):2069-2074. doi:10.1161/01.ATV.0000143936. 77094.a4

29. Salcedo R, Ponce ML, Young HA, et al. Human endothelial cells express CCR2 and respond to MCP-1: direct role of MCP-1 in angiogenesis and tumor progression. Blood. 2000;96(1):34-40.

30. Shi W, Mei H, Deng J, et al. A tissue factor targeted nanomedical system for thrombi-specific drug delivery. Biomaterials. 2012;33(30): 7643-7654. doi:10.1016/j.biomaterials.2012.06.094

31. Getz GS, Reardon CA. Animal models of atherosclerosis. Arterioscler Thromb Vasc Biol. 2012;32(5):1104-1115. doi:10.1161/ATVBAHA. 111.237693

32. Taylor A, Shaw LJ, Fayad Z, et al. Tracking atherosclerosis regression: a clinical tool in preventive cardiology. Atherosclerosis. 2005;180(1): 1-10. doi:10.1016/j.atherosclerosis.2004.12.024

33. Danhier F, Ansorena E, Silva JM, Coco R, Le Breton A, Préat V. PLGAbased nanoparticles: an overview of biomedical applications. J Control Release. 2012;161(2):505-522. doi:10.1016/j.jconrel.2012.01.043

34. Vandervoort J, Ludwig A. Biocompatible stabilizers in the preparation of PLGA nanoparticles: a factorial design study. Int J Pharm. 2002; 238(1-2):77-92.

35. Zhang B, Wang H, Liao Z, et al. EGFP-EGF1-conjugated nanoparticles for targeting both neovascular and glioma cells in therapy of brain glioma. Biomaterials. 2014;35(13):4133-4145. doi:10.1016/j. biomaterials.2014.01.071 
36. Breitenstein A, Tanner FC, Luscher TF. Tissue factor and cardiovascular disease: quo vadis? Circ J. 2010;74(1):3-12.

37. Camera M, Toschi V, Brambilla M, et al. The role of tissue factor in atherothrombosis and coronary artery disease: insights into platelet tissue factor. Semin Thromb Hemost. 2015;41(7):737-746. doi:10.1055/ s-0035-1564041

38. Libby P, Ridker PM, Hansson GK. Progress and challenges in translating the biology of atherosclerosis. Nature. 2011;473(7347):317-325. doi:10.1038/nature 10146
39. Chen D, Xia M, Hayford C, et al. Expression of human tissue factor pathway inhibitor on vascular smooth muscle cells inhibits secretion of macrophage migration inhibitory factor and attenuates atherosclerosis in ApoE -/-mice. Circulation. 2015;131(15):1350-1360. doi:10.1161/ CIRCULATIONAHA.114.013423

40. Zhang J, Zu Y, Dhanasekara CS, et al. Detection and treatment of atherosclerosis using nanoparticles. Wiley Interdiscip Rev Nanomed Nanobiotechnol. 2017;9(1):e1412. doi:10.1002/wnan.1412

\section{Publish your work in this journal}

The International Journal of Nanomedicine is an international, peerreviewed journal focusing on the application of nanotechnology in diagnostics, therapeutics, and drug delivery systems throughout the biomedical field. This journal is indexed on PubMed Central, MedLine, CAS, SciSearch ${ }^{\circledR}$, Current Contents ${ }^{\circledR} /$ Clinical Medicine,
Journal Citation Reports/Science Edition, EMBase, Scopus and the Elsevier Bibliographic databases. The manuscript management system is completely online and includes a very quick and fair peer-review system, which is all easy to use. Visit http://www.dovepress.com/ testimonials.php to read real quotes from published authors. 